\title{
Endoureterotomy is not a sufficient treatment for intrinsic ureteral endometriosis
}

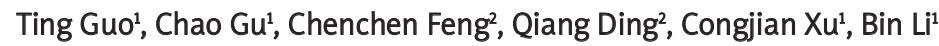 \\ 1Department of Gynaecology, Obstetrics and Gynaecology Hospital, Fudan University, China \\ 2Department of Urology, Huashan Hospital, Fudan University, China
}

\begin{abstract}
Aim: To investigate whether intrinsic ureteral endometriosis could be managed by laser endoureterotomy. Material and methods: We studied retrospectively 6 patients with intrinsic ureteral endometriosis who underwent laser endoureterotomy and reviewed their clinical data. Pathological sections of them have also been studied by immunohistochemistry for expressional levels of oestrogen (ER) and progesterone (PR) receptors. Ten sections of normal endometrium were included as a control.

Results: Five patients had recurrence of ureteral stricture within 6 months postoperatively despite hormonal therapy for 3 to 6 months. One patient had recurrence 8 months after endoureterotomy. Two patients had secondary surgery for ureteroureterostomy and pathology confirmed recurrence of endometriosis. Immunohistochemistry revealed decreased ER and PR expression compared to the control.

Conclusions: Endoureterotomy with hormonal therapy may not be suitable for ureteral endometriosis due to inadequate cutting and expressional change of ER and $P R$.
\end{abstract}

Key words: ureteral endometriosis, endoureterotomy, oestrogen receptor, progesterone receptor.

\section{Introduction}

Among all endometriosis, ureteral endometriosis makes up only $1 \%$ of cases $[1,2]$. Nonetheless, this rare entity can cause severe urological problems due to mechanistic obstruction [3]. For those severe cases, association with deep endometriosis and rectovaginal involvement is often present $[1,4]$. The extrinsic subtype is more commonly seen and causes symptoms by external compression. The intrinsic type, on the other hand, infiltrates the muscularis and directly occludes the ureteral lumen $[4,5]$. Current surgical management of ureteral endometriosis depends on the onset pattern and renal function compromise. The rarity of the disease has made the surgery tailor-made with limited information concerning surgical outcome $[4,5]$.

Whilst it is rational for ureteroureterostomy in extrinsic or mixed cases, laser endoureterotomy in combination with hormonal therapy can be an option for intrinsic subtypes. Thus far there has been no report pertaining to endoureterotomy as a treatment for ureteral endometriosis. Yet as we investigate retrospectively, endoureterotomy may not be optimal due to imbalance of expressions of progesterone receptors (PR) and oestrogen receptors (ER). 


\section{Aim}

In the current study, we aimed to include cases of intrinsic ureteral endometriosis treated by laser endoureterotomy and hormonal therapy, to reveal the surgical outcomes and to discuss the possible reasons in terms of ER and PR expression.

\section{Material and methods}

\section{General information}

A total of 6 samples of unilateral intrinsic ureteral endometriosis and 10 samples of normal uterine endometrium were included in this study. Samples from ureteral endometriosis were all acquired from ureteroscopic cup biopsy followed by laser endoureterotomy and $F 7$ stent implantation from 2000 to 2012. Healthy controls were obtained from hysterectomy due to uterine myoma, all operated at the Obstetrics and Gynaecology Hospital from 2010 to 2011. The study was approved by the local ethical committee and informed consent was obtained for each patient.

\section{Endoureterotomy and follow-ups}

General anaesthesia was performed. A 0.035 inch guidewire was introduced via which the ureteroscope was passed through the ureteral orifice. The Storz semi-rigid ureteroscope was applied in all cases. A cup biopsy was applied when the mass was met and an F 4.5 stent (Optimed, Ettlingen, Germany) was left in each patient (Photo 1). One week later, when

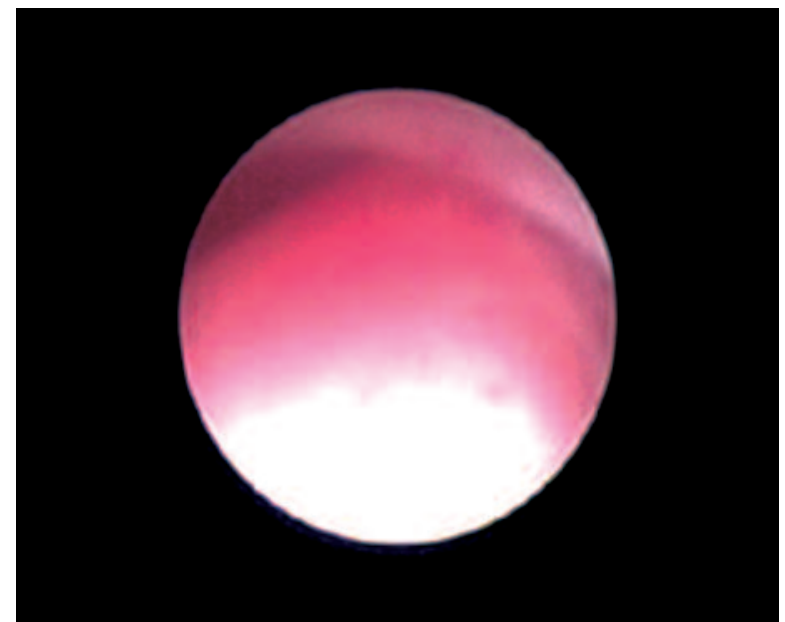

Photo 1. Intraoperative ureteroscopic view of the ureteral mass which was later pathologically confirmed to be intrinsic ureteral endometriosis the pathology confirmed the diagnosis of ureteral endometriosis, patients underwent an endoureterotomy with laser delivery (Luminas, Coherent VersaPulse, Santa Clara, CA, USA) through a 365or 200- $\mu \mathrm{m}$ fibre at the starting energy of $1 \mathrm{~J}, 10 \mathrm{~Hz}$. A retrograde cutting pattern was adopted by slowly advancing the ureteroscope proximally. The incision began at roughly $0.5 \mathrm{~cm}$ below the mass margin and extended over the obstruction for another $0.5 \mathrm{~cm}$. The ureteroscope could be used to assist the procedure by mild lateral parting of the cutting edges. Haemorrhage encountered was cauterized by defocused laser beam and the incision was deepened until the periureteral fat was seen. An F 7 stent was left indwelling for 4 to 8 weeks with urethral catheterization of 1 to 2 days.

Follow-ups included urological and gynaecological outcomes. Patients were referred to gynaecologists for the danazol or gonadotropin-releasing hormone analogue $(\mathrm{GnRH}-\mathrm{a})$ regime. Three months after ureteral stent removal, the patients underwent renogram to assess split renal function. All patients had a pyelogram 6 months post-operatively to re-evaluate the stenosis.

\section{Immunohistochemistry}

Before the enzyme-labelled study, all sections were evaluated by haematoxylin and eosin $(\mathrm{H}+\mathrm{E})$ staining. All samples were formalin-fixed and paraffin-embedded. Endogenous peroxidase of deparaffinized sections was blocked through incubation with 3\% hydrogen peroxide for $15 \mathrm{~min}$. Non-immunized rabbit serum was applied thereafter for $5 \mathrm{~min}$ in order to block non-specific antigen sites. Primary antibodies of rabbit anti-human PR (Ascend Biotechnology Co., Guangdong, China) and ER (Beyotime, Jiangsu, China) were applied for $1 \mathrm{~h}$ each at a dilution of 1 : 100. Following incubation, the sections were extensively rinsed and secondary antibodies were applied accordingly. A DAB (diaminobenzidine tetrahydrochloride) solution was then used for colour developing. Finally, all slides were counterstained with Mayer's haematoxylin blue in $0.3 \%$ ammonia. We used sections of breast cancer and endometrial cancer for positive controls of ER and PR respectively. Sections applied with PBS (phosphate buffer solution) instead of primary antibody were studied as the control. All samples were evaluated semi-quantitatively. For both ER and PR, the extent was graded as 
follows: 0 for $0-5 \%$ of tumour cells stained, 1 for $6-20 \%$ of cells stained, 2 for $21-50 \%$ of cells stained and 3 for $>50 \%$ of cells stained. Intensity of staining was graded as follows: 1 for light yellow, 2 for dark yellow and 3 for brown. The final value, calculated by the sum of the two scores, was as follows: 0 for negative (1-2), 1 for mild (3), 2 for moderate (4), and 3 for severe (5-6) [6-9].

\section{Statistical analysis}

SPSS 17.0 for Windows was utilized for statistical analyses. All data were presented as mean \pm standard deviation. Student's t-test was applied for comparison between two groups.

\section{Results}

The average age of the patients in the case group was $46.22 \pm 4.5$ years. None of the patients in either group had menopause. Preoperative renogram revealed impaired renal function in the ipsilateral kidney and all 6 patients in the case group has obstructions in the distal ureters. Four patients had the disease on the left side. Among the 6 patients who were incised and drained adequately intra- and postoperatively, five had recurrence of ureteral stenosis 6 months postoperatively and 1 patient had recurrence in the $8^{\text {th }}$ month postoperatively. Three of them received the hormonal therapy for 3 months and the other 3 had the therapy for 6 months. Two patients eventually underwent open surgery to have the stricture removed and pathology confirmed the recurrence of endometriosis.

Staining of both ER and PR was located in the nucleus. $\mathrm{H}+\mathrm{E}$ staining revealed that ureteral endometriosis had identical morphological features with normal endometrium (Photo 2 A). Nonetheless, ex- pression of both $\mathrm{PR}$ and ER was significantly lower than the normal control (Table I, Photos 2 B and 2 C).

\section{Discussion}

Endometriosis indicates the presence of endometrial glands and stroma outside the uterus. It is a common disease affecting women between menarche and menopause. Involvement of the urinary tract occurs only in $1 \%$ to $2 \%$ of cases and frequencies for bladder, ureter and kidney are $40: 5: 1$. Almost half of the patients with ureteral endometriosis are asymptomatic at diagnosis and up to $47 \%$ of patients require nephrectomy due to non-functioning kidney or suspicion of a ureteral urothelial carcinoma. Most commonly, the disease affects the unilateral side and distal segment of the ureter. It is however very rare for upper segments of the ureter to be involved [10].

The goal of treatment for ureteral endometriosis is to relieve obstruction and preserve as much renal function as possible. In most cases, management involves participation of multi-disciplinary cooperation [3]. Although there is thus far no standardized regime for treatment, a combination of surgical intervention followed by hormonal therapy is recommended. Single therapy with hormone is indicated for early-stage disease and not for advanced disease

Table I. Comparison of PR and ER expression between ureteral endometriosis and normal endometrium

\begin{tabular}{|lccc|}
\hline Variables & $N$ & PR & ER \\
\hline Ureteral endometriosis & 6 & $0.7 \pm 0.5$ & $0.6 \pm 0.4$ \\
\hline Normal endometrium & 10 & $2.0 \pm 0.8$ & $2.1 \pm 0.9$ \\
\hline Value of $p$ & & 0.0032 & 0.0019 \\
\hline
\end{tabular}

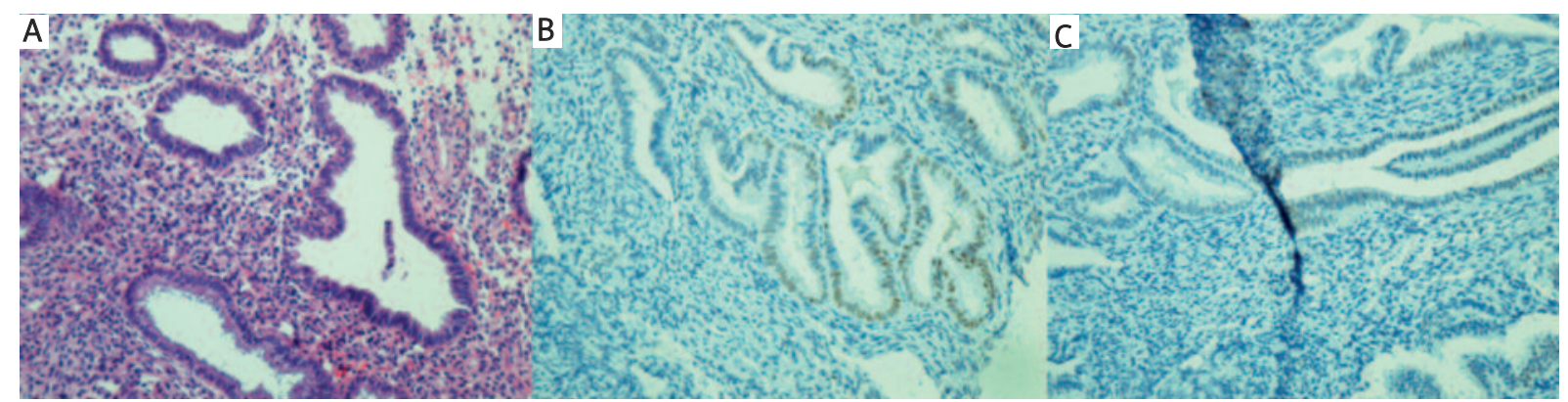

Photo 2. Pathological sections of ureteral endometriosis demonstrating: A - features of normal endometrium in $\mathrm{H}+\mathrm{E}$ staining and marginal staining of both, $\mathrm{B}$ - progesterone receptors (PR) and C- oestrogen receptors (ER), all captured at $200 \times$ magnification 
due to high recurrence and the limited effect on scar tissue. In combination with total abdominal hysterectomy with bilateral salpingo-oophorectomy, the therapy can decrease the recurrence from $27 \%$ to $3 \%$ [11]. Current therapy includes danazol, GnRH agonist (leuprolide, goserelin), medroxyprogesterone, oestrogen-progestin combination and progestin alone [12-14]. Danazol and GnRH are most commonly used, with the mechanism of antagonizing the effect of gonadotropin, entailing the eventual effect of inhibition of ovarian function. Most authors have reported use of these medications for ureteral endometriosis yet the long-term outcome is still lacking [15].

It is interesting that surgical interventions for ureteral endometriosis have not yet involved endoureterotomy, or at least there are no such reports. Since the incidence for intrinsic type is very rare, we can only document 5 cases which were treated endoscopically. The goal of surgery is to remove or relieve obstruction since the scar tissue does not respond to hormonal therapy. Laparoscopic ureteroureterostomy and ureterolysis are most commonly practised [7, 16-18]. The success rate reported by ureteroscopic balloon dilation has been satisfactory in short-term follow-ups [19]. Another ureteroscopic approach achieved short-term success by alloy stent implantation [20]. Generally, attempts with endoscopic treatment are based on the remediability of renal function and have no effect on the underlying aetiology.

In the current study, we investigated the efficacy of endoureterotomy in combination with postoperative hormonal therapy and found that the relief does not last as long as we expected. In the immunohistochemical study, we identified significant decreased expression of both ER and PR in comparison to the normal endometrium, which conforms to previous studies pertaining to endometriosis in other sites [21]. The insufficiency of endoureterotomy may stem from: 1 . inadequate incision of the endometrial tissue. Since the intrinsic and extrinsic types of ureteral endometriosis may coexist, endoscopic cutting may overlook external endometrium. Also, due to the risk of potential ureteral avulsion and laceration, longitudinal ablation may be compromised in cutting the endometrium in the muscularis. 2. Due to expressional decrease in both ER and PR in the ectopic endometrium, the response to hormonal therapy may be compromised and in fact we have observed the phenomenon that postoperative hormonal consolidation cannot prevent recurrence. Ureteroure- terostomy should be considered in case of intrinsic ureteral endometriosis.

Our study has limitations. The retrospective nature and the rarity of the disease indicate that the results should be interpreted carefully. Loss of both $E R$ and $P R$ in intrinsic ureteral endometriosis reflects the nature of refractory deep endometriosis, which requires more complete resection with hormonal replacement consolidation. Last but not least, due to the rarity of the disease and lack of unified treatment guidelines, patients were prone to start with less invasive therapies even though they were warranted that secondary surgery could be more sophisticated given a prior endoureterotomy. Our results at the very least demonstrate non-superiority of endoureterotomy compared with ureteroureterostomy. Given the fact that endopyelotomy can hardly reach the success rate of open pyeloplasty, it is reasonable not to practise endoureterotomy in ureteral endometriosis unless otherwise indicated [17].

\section{Conclusions}

Although rare, intrinsic ureteral endometriosis can cause silent renal failure. We investigated the efficacy of endoureterotomy plus hormonal therapy. The results indicate that the combination still entails recurrence of endometriosis. Decrease of ER and PR expression and inadequate cutting are probably responsible. Intrinsic ureteral endometriosis should be managed by ureteroureterostomy.

\section{Acknowledgments}

Ting Guo and Chao Gu - equal contributors.

\section{References}

1. Wang H, Gorpudolo N, Li Y, et al. Elevated vascular endothelia growth factor-A in the serum and peritoneal fluid of patients with endometriosis. J Huazhong Univ Sci Technolog Med Sci 2009; 29: 637-41.

2. Stillwell TJ, Kramer SA, Lee RA. Endometriosis of ureter. Urology 1986; 28: 81-5.

3. Langebrekke A, Qvigstad E. Ureteral endometriosis and loss of renal function: mechanisms and interpretations. Acta Obstet Gynecol Scand 2011; 90: 1164-6.

4. Chapron C, Chiodo I, Leconte M, et al. Severe ureteral endometriosis: the intrinsic type is not so rare after complete surgical exeresis of deep endometriotic lesions. Fertil Steril 2010; 93: 2115-20.

5. Mereu L, Gagliardi ML, Clarizia R, et al. Laparoscopic management of ureteral endometriosis in case of moderate-severe hydroure teronephrosis. Fertil Steil 2010; 93: 46-51. 
6. Feng C, Guan M, Ding Q, et al. Expression of pigment epitheliumderived factor in bladder tumour is correlated with interleukin8 yet not with interleukin-1alpha. J Huazhong Univ Sci Technolog Med Sci 2011; 31: 21-5.

7. Feng CC, Ding Q, Zhang YF, et al. Pigment epithelium-derived factor expression is down-regulated in bladder tumors and correlates with vascular endothelial growth factor and matrix metalloproteinase-9. Int Urol Nephrol 2011; 43: 383-90.

8. Feng C, Wu Z, Guo T, et al. BLCA-4 expression is related to MMP-9, VEGF, IL-1alpha and IL-8 in bladder cancer but not to PEDF, TNF-alpha or angiogenesis. Pathol Biol (Paris) 2012; 60: e36-40.

9. Feng CC, Wang PH, Guan M, et al. Urinary BLCA-4 is highly specific for detection of bladder cancer in Chinese Han population and is related to tumour invasiveness. Folia Biol (Praha) 2011; 57: 242-7.

10. Rosemberg SK, Jacobs H. Endometriosis of the upper ureter. J Urol 1979; 121: 512.

11. Takeuchi S, Minoura H, Toyoda N, et al. Intrinsic ureteric involvement by endometriosis: a case report. J Obstet Gynaecol Res 1997; 23: 273

12. Zanetta G, Webb M, Segura JW. Ureteral endometriosis diagnosed at ureteroscopy. Obstet Gynecol 1998; 91: 857.

13. Lavelle KJ, Melman AW, Cleary RE. Ureteral obstruction owing to endometriosis: reversal with synthetic progestin. J Urol 1976; 116: 665.

14. Pittaway DE, Daniell JF, Maxson WS, et al. Recurrence of ureteral obstruction caused by endometriosis after danazol therapy. Am J Obstet Gynecol 1982; 143: 720.

15. Matsuura K, Kawasaki N, Oka M, et al. Treatment with danazol of ureteral obstruction caused by endometriosis. Acta Obstet Gynecol Scand 1985; 64: 339.

16. el Kahder K, Guille F, Patard JJ, et al. Ureteral reimplantation on psoas bladder: long-term results. Acta Urol Belg 1998; 66: 15.

17. Wu Z, Feng C, Ding Q, et al. Ureteroscopic holmium:YAG laser endopyelotomy is effective in distinctive ureteropelvic junction obstructions. Videosurgery Miniinv 2011; 6: 144-9.

18. Kupajski M, Tkocz M, Ziaja D. Modern management of stone disease in patients with a solitary kidney. Videosurgery Miniinv 2012; 7: 1-7.

19. Vercellini P, Pisacreta A, Pesole A, et al. Is ureteral endometriosis an asymmetric disease? BJOG 2000; 107: 559.

20. Kulkarni RP, Bellamy EA. A new thermo-expandable shape-memory nickel-titanium alloy stent for the management of ureteric strictures. BJU Int 1999; 83: 755.

21. Engemise SL, Willets JM, Taylor AH, et al. Changes in glandular and stromal estrogen and progesterone receptor isoform expression in eutopic and ectopic endometrium following treatment with the levonorgestrel-releasing intrauterine system. Eur J Obstet Gynecol Reprod Biol 2011; 157; 101-6.

Received: 7.08.2012, accepted: 2.02.2013. 